\title{
Proporción sexual, relación talla-peso y distribución geográfico-batimétrica de Squalus cubensis (SQUALIFORMES: SQUALIDAE) en la costa Caribe de Centroamérica
}

\author{
Sex Ratio, Length-Weight Relationship and Geographic-Bathymetric Distribution of \\ Squalus Cubensis (SQUALIFORMES: SQUALIDAE) in the \\ Caribbean Coast of Central America
}

\author{
Rosario Benavides-Morera \\ rbmorera@gmail.com \\ Escuela de Ciencias Biológicas, Universidad Nacional \\ Laboratorio de Oceanografía y Manejo Costero, Universidad Nacional \\ Heredia, Costa Rica \\ Carlos Luis Brenes-Rodríguez \\ cbrenes.una@gmail.com \\ Laboratorio de Oceanografía y Manejo Costero, Universidad Nacional \\ Heredia, Costa Rica \\ Amaru Márquez-Artavia \\ tupac.marquez@gmail.com \\ Laboratorio de Oceanografía y Manejo Costero, Universidad Nacional \\ Heredia, Costa Rica
}

Recibido-Received: 13/dic/2015 / Corregido-Corrected: 13/set /2016.

Aceptado-Accepted: 17/set/2016 / Publicado-Published: 31/jul /2017.

\begin{abstract}
Resumen
Se describe la distribución geográfica y batimétrica de Squalus cubensis capturados a bordo del B/O Miguel Oliver en la costa Caribe de Centroamérica, durante una campaña de prospección pesquera realizada del 3 de enero hasta el 3 de febrero del 2011. Se analizaron 96 lances, se capturaron 140 individuos (65 machos y 75 hembras) de 24 hasta $100 \mathrm{~cm}$ de longitud total, y entre 70 y 3040 grs de peso total. En cuanto a la talla, se encontró una marcada diferencia que indica dimorfismo sexual. La relación talla-peso para ambos sexos no presentó diferencias significativas en las pendientes de las regresiones entre sexos. La distribución espacial de las capturas mostró que la mayor cantidad de individuos se obtuvo, principalmente, en Panamá a profundidades que van desde los 250 hasta los $950 \mathrm{~m}$, seguido por Honduras. Más del $75 \%$ de las capturas se dieron en un estrato de la columna de agua con temperaturas entre los $17^{\circ} \mathrm{C}$ y los $12^{\circ} \mathrm{C}$. El presente trabajo proporciona información preliminar importante, pues es la primera vez que se documenta una captura de esta especie que permita estimar proporción de sexos, estructuras de tallas y la relación peso-talla de S. cubensis en el Caribe de Centroamérica.
\end{abstract}

Palabras claves: Tiburón; mar Caribe; América Central; temperatura; batimetría. 
UNICIENCIA Vol. 31, No. 2, pp. 69-82. Julio-diciembre, 2017.

ISSN Electrónico: 2215-3470

URL: www.revistas.una.ac.cr/uniciencia

DOI: http://dx.doi.org/10.15359/ru.31-2.5

Email: revistauniciencia@una.cr

\begin{abstract}
This article describes the geographic and bathymetric distribution of Squalus cubensis; some specimens were captured during a prospecting survey campaign that was held from January 3 to February 3, 2011, aboard the R/V Miguel Oliver on the Caribbean coast of Central America. 140 individuals were captured in 96 sets (65 males and 75 females) from 24 to $100 \mathrm{~cm}$ in total length, and between 70 and 3,040 grs of total weight. A marked difference in the sizes was found, indicating a sexual dimorphism. The length-weight relationship between sexes showed no significant differences in the slopes of the respective regressions. The spatial distribution of catches showed that more individuals were obtained primarily in Panama at depths ranging from 250 to $950 \mathrm{~m}$, followed by Honduras. Over $75 \%$ of the catch occurred in a layer of the water column with temperatures between $12^{\circ} \mathrm{C}$ and $17^{\circ} \mathrm{C}$. This study provides important preliminary information about sex ratio and lengthweight relationship in $S$. cubensis captured in this area.
\end{abstract}

Keywords: Shark; Caribbean Sea; Central America; temperature; bathymetry.

El Caribe de América Central se extiende desde Belice hasta Panamá y su costa tiene muchas características distintivas, esta es considerada como un margen pasivo con unas pocas formaciones rocosas elevadas (Cortés, 2007). Las principales corrientes en el Caribe van de este a oeste, y el istmo centroamericano desvía parte de esos flujos hacia el sur a lo largo de Nicaragua, Costa Rica y Panamá. El resto de las corrientes continúan al oeste hacia Honduras y luego se curvan hacia el norte en Belice (Jackson y D'croz, 1997). Belice posee una plataforma continental en la cual se ubica un ecosistema complejo formado por el arrecife más grande en el océano Atlántico y tres atolones en sus $457 \mathrm{~km}$ de línea costera (FAO, 2006). Honduras se distingue por poseer una gran plataforma continental de $53500 \mathrm{~km}^{2}$ adyacente a la de Nicaragua, la cual es bastante ancha y se vuelve angosta conforme se dirige hacia el sur donde empieza la de Costa Rica, la cual se caracteriza por ser sumamente angosta, para finalmente extenderse durante $1288 \mathrm{~km}$ en los márgenes de la costa Caribe de Panamá (Aㅇ, 2006).

El orden de los Squaliformes está constituido por seis familias, una de ellas es la familia Squalidae, esta, a su vez, incluye 24 géneros y al menos 97 especies (Nelson, Grande y Wilson, 2016). Contiene tiburones pequeños y de gran talla, de los cuales los más diversos se encuentran en aguas profundas. La mayoría de las especies de Squaliformes tienen estructuras poblacionales complejas. Una característica común de este grupo de peces es la segregación temporal y espacial por sexo, talla y condición de madurez (Braccini, Gillanders y Walker, 2006).

Squalus cubensis (Rivero, 1936) se caracteriza por poseer un cuerpo cilíndrico, carece de aleta anal, tienen hocico corto y dos aletas dorsales con el extremo negro con una espina larga, además, el margen posterior de las pectorales muy cóncavo (Compagno, 1984).

Su distribución geográfica corresponde al océano Atlántico occidental subtropical, desde Carolina del Norte hasta Florida. También vive en la parte norte del Golfo de México y en aguas caribeñas cercanas a Cuba, República Dominicana y Haití (Compagno, 1984). Se menciona también ocasionalmente en Panamá, Nicaragua y Honduras (Monzini, 2006). En las costas de América del Sur se le ha reportado en trabajos de Brasil y Argentina. Muy recientemente el género Squalus de la región sur oeste del océano Atlántico ha sido revisado a nivel taxonómico y morfológico, debido a grandes dificultades de diferenciación entre especies (Viana, Carvalho y Gomes, 2016). Es evidente, entonces, que esta especie ahora no ofrece un vasto y amplio rango de distribución geográfica como se suponía en un principio al describir su distribución, sino que 
posterior a la evidencia anteriormente mencionada, esta es mucho más restringida. En cuanto a su distribución batimétrica Espinosa, Castro-Aguirre y Huidobro (2004) lo ubican entre los 50 y $400 \mathrm{~m}$ de profundidad.

Se le ha reportado previamente en el Golfo de México (Castillo-Géniz et al., 1998) en donde ocasionalmente forma parte de las capturas artesanales en la pesquería de tiburones. Márquez (2002) lo cataloga como una especie de la cual existe muy poca información en México. Este tiburón también forma parte de la captura incidental de la pesca de atún en las costas atlánticas de Venezuela (Arocha, F, Arocha y Marcano, 2002). Kiraly, Moore y Jasinski, (2003) lo ubican dentro de la zona económica exclusiva de los Estados Unidos, en los alrededores de la isla de Puerto Rico y de las Islas Vírgenes. Álvarez-León y Rey-Carrasco (2003), ubican a S. cubensis por primera vez en el Caribe de Colombia en un crucero realizado en 1979. En Argentina y Uruguay forma parte de una lista actualizada de condrictios (Menni y Lucifora, 2007). Páramo, Wolff y Saint-Paul (2012) incluye S. cubensis como parte de la ictiofauna de profundidad del norte de Colombia. Estos trabajos anteriormente citados dan información sobre su distribución geográfica; sin embargo, no existen estudios previos sobre la estructura de sus poblaciones en el Caribe de Centroamérica.

La vasta escala geográfica de ecosistemas marinos pelágicos limita la habilidad de monitorear las poblaciones de tiburones adecuadamente. Así, el efecto de la explotación sobre los tiburones en muchas poblaciones se mantiene incierto. Su manejo y su conservación se han visto obstaculizados por la falta del conocimiento de su estado o incluso por la dirección de la tendencia de la población (Baum et al., 2003).

En general para el Caribe centroamericano se tiene documentada muy poca información biológica de especies, a excepción de Belice y algunas secciones de la costa de Panamá que sí disponen de esta (Alvarado, Solís-Marín y Ahearn, 2008). En este sentido, existe escaso conocimiento biológico y pesquero de la especie en estudio, parte de ello puede deberse a que esta es habitual en hábitats profundos. Esta realidad reviste de un valor adicional los resultados presentados en este trabajo, el cual pretende aportar información novedosa en cuanto a algunos aspectos biológicos-pesqueros relacionados con las capturas de S. cubensis en esta área.

\section{Metodología}

Área de estudio, arte y faenas de pesca: El área de estudio abarcó la plataforma y el talud continental del Caribe Centroamericano, desde el norte de Belice (Latitud 17²4.34 N, Longitud $87^{\circ} 75.80 \mathrm{~W}$ ) hasta el sur de Panamá (Latitud $08^{\circ} 57.652 \mathrm{~N}$, Longitud $77^{\circ} 26.880 \mathrm{~W}$ ). En la plataforma continental frente a las costas de Nicaragua no se realizaron transeptos debido a que no se contó con el aval gubernamental para muestrear esa zona. Además, en el sur de Honduras existe una gran zona protegida en la cual no es permitido realizar este tipo de investigación (ver figura 1).

La faena de pesca se realizó entre el 3 de enero y el 3 de febrero del 2011 a bordo del buque oceanográfico Miguel Oliver, el cual cuenta con una red de arrastre tipo LOFOTEN para fondos duros (arrastre con puertas, con 2 tapas de paño polietileno 48,7 m de longitud, copo de 14,3 m de largo y $35 \mathrm{~mm}$ de luz de malla de poliamida y tren de arrastre de 41,7 $\mathrm{m}$ de longitud de 1000 Kg de peso aproximado).

El estudio se desarrolló en la plataforma y talud continental desde los 100 hasta los 1500 $\mathrm{m}$ de profundidad. Previamente se establecieron 25 transeptos equidistantes y perpendiculares 
a la costa, cubriendo el área total de estudio (ver figura 1). Se realizaron lances (entre 5-6 por transepto) durante el día, con una duración de 30 min de arrastre efectivo, se contó desde el momento del firme del lance en el fondo hasta el inicio de la orden de virada. El control del tiempo de arrastre se realizó mediante el empleo de los sensores de red (ITI-SCANMAR).

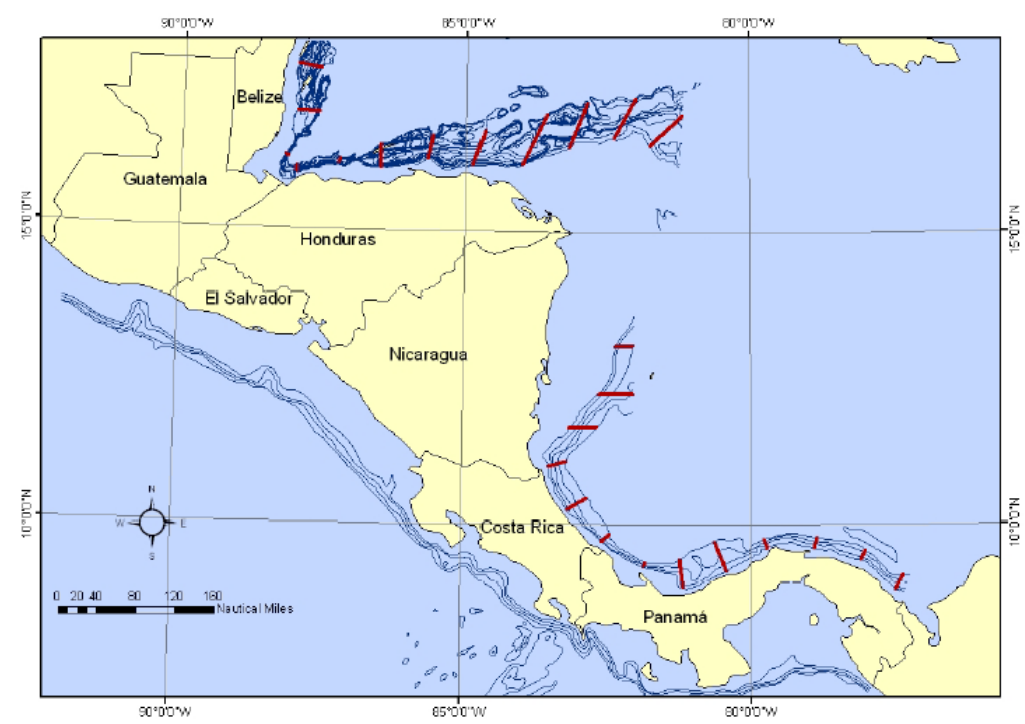

Figura 1. Área de estudio que indica los transeptos equidistantes y perpendiculares a la línea de costa. Datos propios del estudio.

Cada espécimen capturado fue medido en su longitud total ( $\mathrm{Lt} \pm 1 \mathrm{~mm}$ ), pesado (Peso total, Pt \pm 1grs) y sexado.

Adicionalmente, se hicieron mediciones de las variables ambientales: temperatura, salinidad y oxígeno disuelto en la columna de agua, con ayuda de un CTD (conductividad, temperatura y profundidad por sus siglas en inglés)

Análisis de datos: Se determinó la distribución de frecuencias de longitud para la totalidad de la muestra y la proporción de sexos, esta se calculó dividiendo el número total de hembras entre el número total de machos, se aplicó la prueba de Xi-cuadrada con el fin de determinar si existe una diferencia significativa entre la proporción de sexos observada y la esperada (1:1), con la ecuación:

donde:

$$
\chi^{2}=\Sigma\left(\frac{O i-E i}{E i}\right)^{2}
$$

$\mathrm{O}_{\mathrm{i}}=$ resultados observados, $\mathrm{E}_{\mathrm{i}}=$ resultados esperados; si $\mathrm{x}^{2}$ calculada $<\mathrm{x}^{2}$ tablas, no es significativamente diferente de $1: 1,0 \mathrm{x}^{2}$ calculada $>\mathrm{x}^{2}$ tablas, si es significativamente diferente de 1:1 (Daniel, 2012).

La caracterización de la estructura de tallas para S. cubensis se basó en el análisis de las capturas. La distribución de tallas por sexo se presentó gráficamente mediante histogramas de frecuencia. Se ajustaron regresiones no lineales entre peso total y longitud total para ambos sexos 
separadamente $(b=3)$, para evaluar si el crecimiento de esta especie es alométrico o isométrico. Se realizó una prueba $t$ para comparar estas regresiones entre machos y hembras, se calculó el coeficiente de determinación $\left(\mathrm{r}^{2}\right)$ para cada regresión. Finalmente, con base en el registro por país y tomando en cuenta la profundidad registrada durante el estudio, se realizó una distribución vertical de la captura de estas especies. Se graficó la frecuencia de lances en función de la profundidad de captura y se compararon las distribuciones verticales de temperatura $\left({ }^{\circ} \mathrm{C}\right)$ para dos transeptos paralelos a la costa, con los lugares en donde las capturas fueron más representativas.

Para el análisis de los datos se utilizaron paquetes informáticos como Excel, Grapher y SURFER 11 (2012). Este análisis se llevó a cabo en el Laboratorio de Oceanografía y Manejo Costero de la Universidad Nacional.

\section{Resultados}

Se analizaron datos de un total de 96 lances efectivos en los que se capturaron 140 individuos de S. cubensis (65 machos y 75 hembras).

Proporción de sexos: Aunque se registró una mayor presencia de hembras respecto a los machos (53.6\% hembras y 46.4\% machos), resultando en una proporción 1:1.15 (macho-hembra), esta proporción de sexos no difiere significativamente de la esperada 1:1 $\left(\mathrm{X}^{2}=0,70\right.$. g.l $\left.=1, \mathrm{P}>0.05\right)$

Estructura de tallas: La captura de esta especie estuvo conformada por individuos con tallas entre 24 y $100 \mathrm{~cm}$ de longitud total $(\mathrm{Lt})$ con una media de $48 \mathrm{~cm}$. La talla varió entre 31 y $57 \mathrm{~cm} \mathrm{Lt}(41 \pm 3.7 \mathrm{~cm})$ en los machos y entre 24 y $100 \mathrm{~cm} \mathrm{Lt}(48 \pm 11.7 \mathrm{~cm})$ en las hembras. Las distribuciones de frecuencias de tallas para machos y hembras fueron diferentes (ver gráfica 2).

En las hembras se observó que la mayor cantidad de individuos (28\%) se encontraban entre $45 \mathrm{~cm}$ y $50 \mathrm{~cm}$ (ver figura 2A), mientras que casi el 60\% de los machos con tallas entre $40 \mathrm{~cm}$ y $45 \mathrm{~cm}$ (ver figura 2B). Se encontró para las tallas máximas una diferencia marcada que indica un dimorfismo sexual entre machos y hembras.

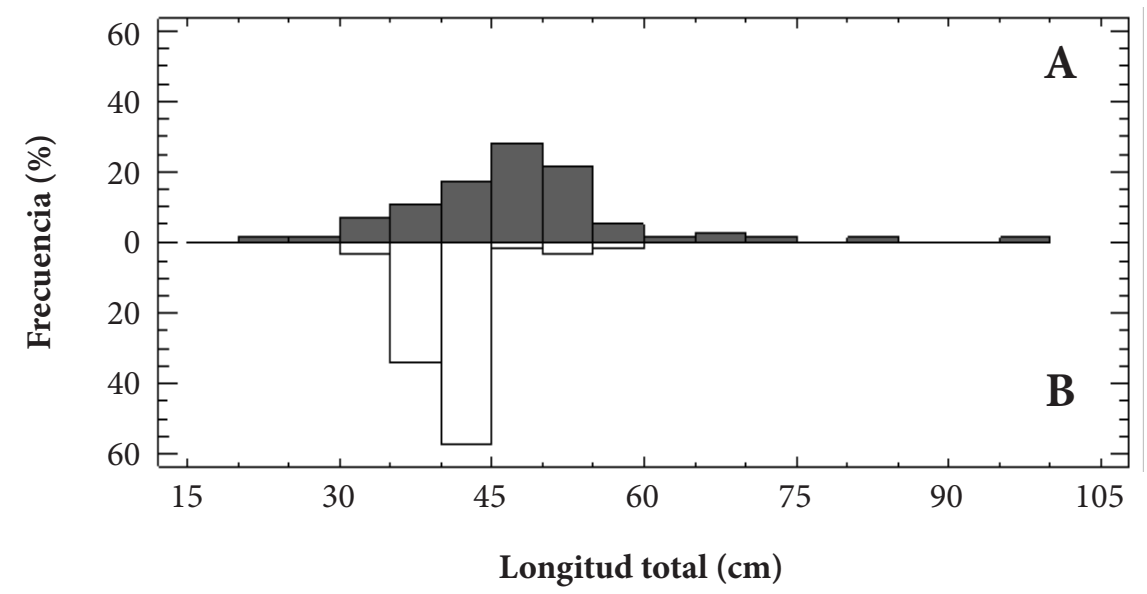

Figura 2. Distribución de la frecuencia de talla (intervalo de clase de $5 \mathrm{~cm}$ ) de (A) hembras y (B) machos de S. cubensis capturados en el Caribe centroamericano. Datos propios del estudio. 
UNICIENCIA Vol. 31, No. 2, pp. 69-82. Julio-diciembre, 2017.

Email: revistauniciencia@una.cr

Relaciones biométricas: El peso total $(\mathrm{Pt})$ de las hembras $(\mathrm{n}=74)$ varió de 70 a $3040 \mathrm{~g}(611.1$ $\mathrm{g} \pm 433.2 \mathrm{~g})$ y el de los machos $(\mathrm{n}=63)$ de 70 a $758 \mathrm{~g}(336.5 \mathrm{~g} \pm 82.8 \mathrm{~g})$.

La relación longitud total - peso mostró un valor de la pendiente para las hembras de 3,087 $\left(\mathrm{a}=0,38 \mathrm{r}^{2}=0,9633\right)$ (ver figura $\left.3 \mathrm{~A}\right)$ y para machos de $2,9925\left(\mathrm{a}=0,36 \mathrm{r}^{2}=0,755\right)$ (ver grafica 3B).

La prueba $t$ mostró que no existen diferencias significativas en las pendientes resultantes tanto para hembras $(t=1,9939, \mathrm{~g} .1=73, \mathrm{p}>0,05)$ como para machos $(t=1,9990, \mathrm{~g} .1=62, \mathrm{p}>0,05)$, con una relación de tipo isométrica en ambos sexos. Esta relación indica que el organismo experimenta un incremento proporcional tanto en peso como en longitud y que este es igual en hembras como en machos.

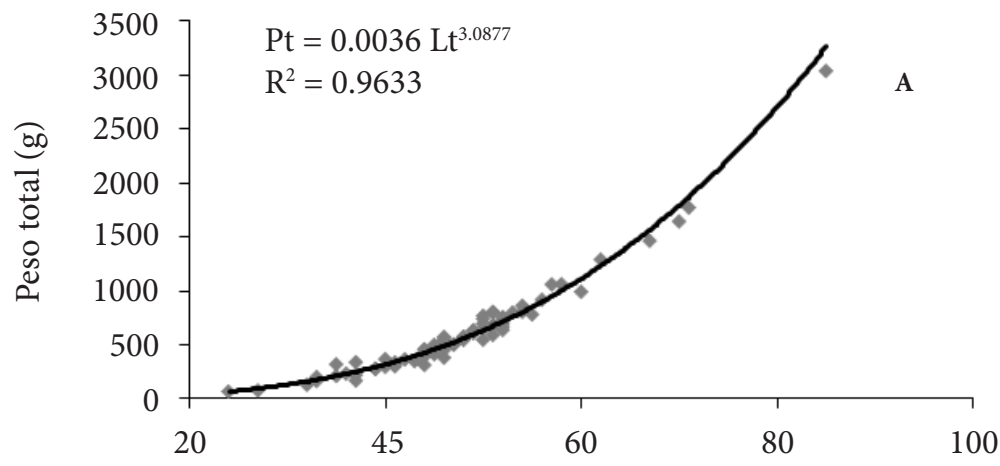

Longitud total $(\mathrm{cm})$

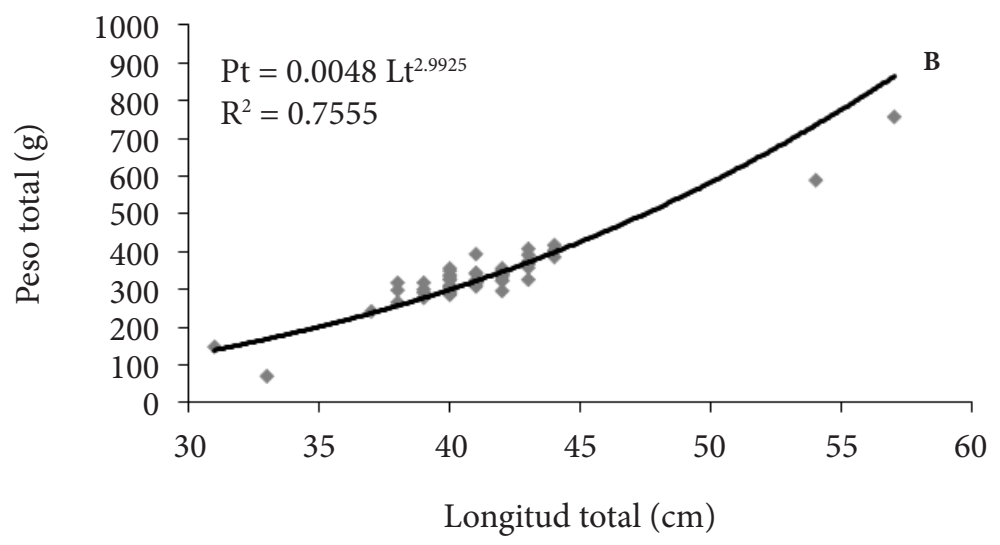

Figura 3. Relación entre la longitud total y el peso total para hembras (A) y machos (B) de S. cubensis capturados en el Caribe centroamericano. Datos propios del estudio. 
Distribución: En la figura 4 se muestra la distribución batimétrica por país, de los ejemplares capturados sobre la plataforma y talud continental del Caribe centroamericano. La mayor cantidad de individuos fueron capturados en Panamá a profundidades que van desde los 250 hasta los $950 \mathrm{~m}$.

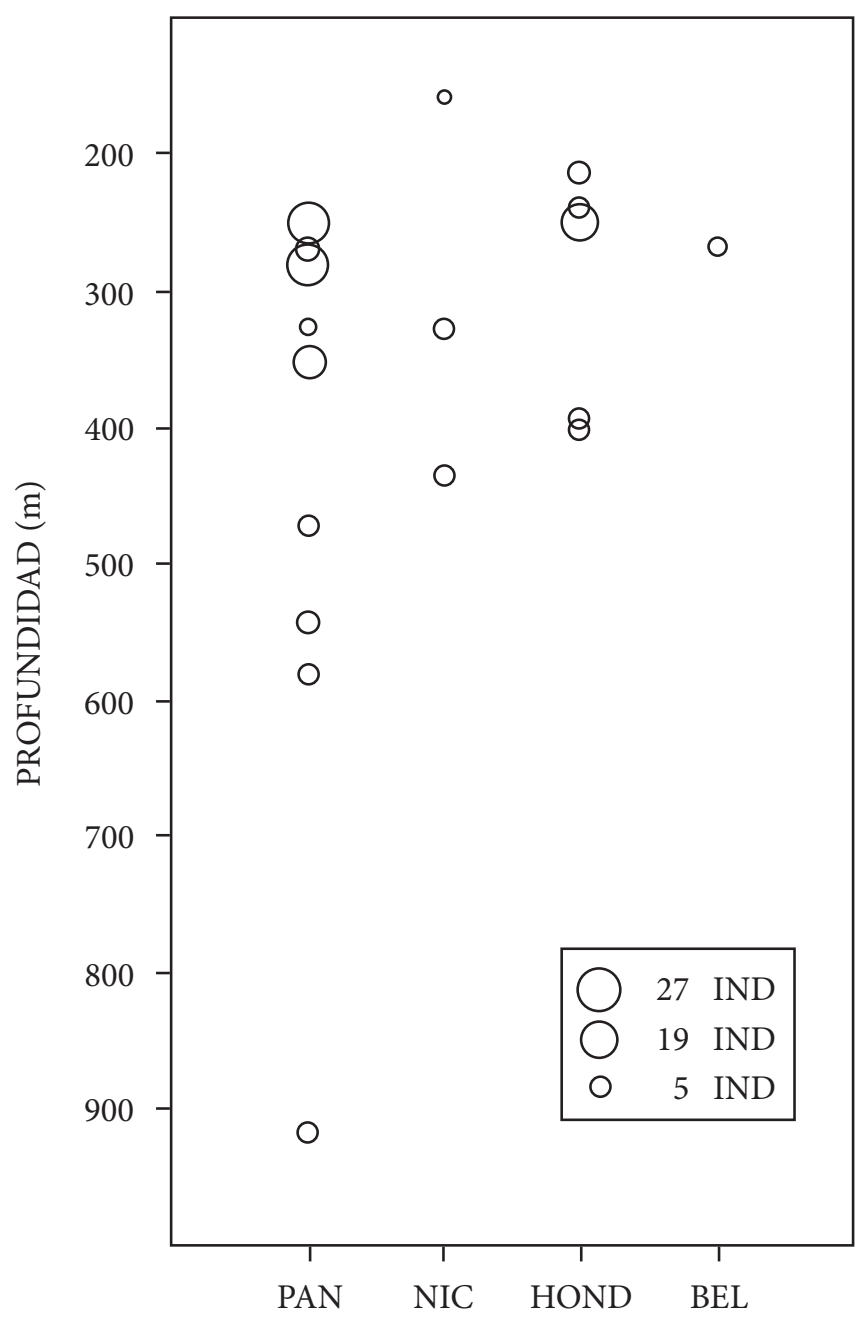

Figura 4. Distribución batimétrica por país de S. cubensis capturados en el B/O Miguel Oliver durante el período de estudio. Datos propios del estudio.

La figura 5 muestra la frecuencia de lances en función de la profundidad de captura. De los 19 lances en que aparecieron especímenes de S. cubensis, el intervalo de profundidad de mayor frecuencia (en más del 75\% de los lances) se ubicó entre los 200 m y $500 \mathrm{~m}$. En términos de número de individuos esto significó que, de un total de 140 especímenes, 123 se capturaron a dichas profundidades. 
UNICIENCIA Vol. 31, No. 2, pp. 69-82. Julio-diciembre, 2017.

URL: www.revistas.una.ac.cr/uniciencia

Email: revistauniciencia@una.cr
ISSN Electrónico: 2215-3470

DOI: $\underline{\text { htp://dx.doi.org/10.15359/ru.31-2.5 }}$

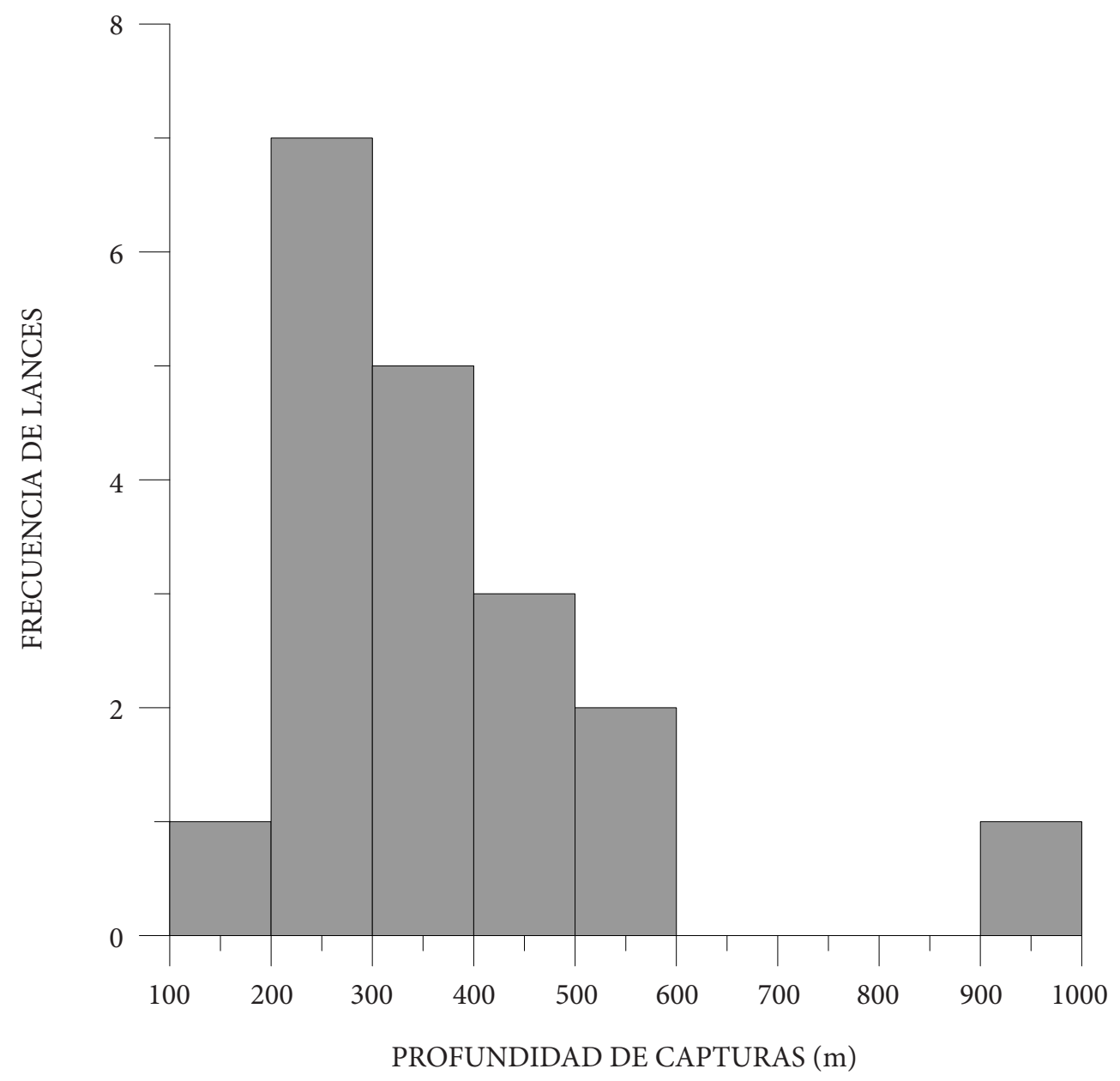

Figura 5. Frecuencia de lances ( $\mathrm{n}=19)$ en función de la profundidad de captura de $S$. cubensis. Datos propios del estudio.

En la figura 6 se presentan las distribuciones verticales de temperatura para dos transeptos paralelos a las costas panameña (6A) y hondureña (6B). Se escogieron estas dos regiones por ser las que presentaron el mayor porcentaje de lances (78\%) con capturas de S. cubensis durante toda la campaña. En ambas áreas se observa una amplia capa de mezcla muy homogénea que se extiende hasta los $75 \mathrm{~m}$ de profundidad con temperaturas superiores a los $26^{\circ} \mathrm{C}$. La termoclina se localiza a una profundidad del orden de los $180 \mathrm{~m}$ (definida por la profundidad de la isoterma de $20^{\circ} \mathrm{C}$ ). 

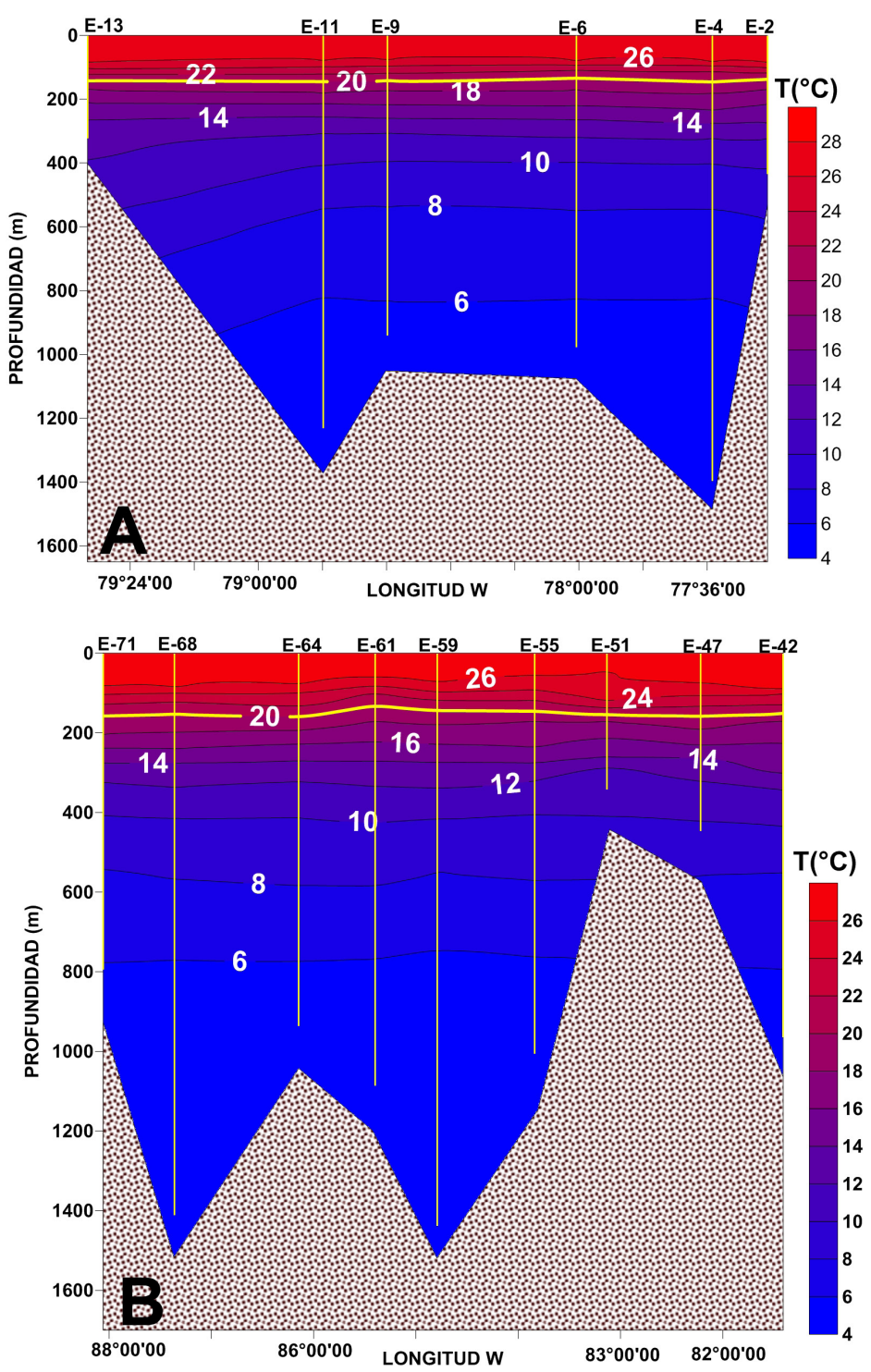

Figura 6. Distribuciones verticales de temperatura $\left({ }^{\circ} \mathrm{C}\right)$ para un transepto paralelo a la costa caribeña de Panamá (A) y de Honduras (B). Datos propios del estudio.

\section{Discusión}

El análisis de proporción de sexos para los individuos capturados indicó que no existe una diferencia significativa en esta característica poblacional dentro de las capturas. Dicho análisis de sexos no se hizo por área ni por profundidad, sino sobre la población total, caso contrario al estudio con trampas y líneas bentónicas hecho por Fischer et al. (2006), donde se encontró una proporción de sexos mayor para hembras que para machos de Squalus mitsukurii. Por lo tanto, por las condiciones de nuestro muestreo, no podría sugerirse que exista una vulnerabilidad a las faenas de pesca en función del sexo, ni tampoco una predominancia por sexo en las poblaciones asociadas a una posible segregación sexual por área o profundidad como lo reportaron Hazin et al. (2006) en un estudio reproductivo para Squalus megalops en el Atlántico sur. 
UNICIENCIA Vol. 31, No. 2, pp. 69-82. Julio-diciembre, 2017.

En cuanto a la estructura de tallas, las hembras resultaron ser más grandes que los machos (figura 2). Lo anterior coincide con lo expuesto por Cortés (2004) y lo encontrado por Yano y Tanaka (1989) para una población de Etmopterus unicolor que también pertenece al orden de los Squaliformes. Para las hembras de S. cubensis se obtuvo una talla máxima de $100 \mathrm{~cm}$ LT, mientras que los machos $57 \mathrm{~cm}$ LT. Estos resultados concuerdan con lo encontrado por Compagno, Dando y Fowler (2005), quienes reportaron una longitud máxima para esta especie de $110 \mathrm{~cm} \mathrm{LT}$, mientras que en la adultez muestra una talla promedio de $75 \mathrm{~cm}$, dato que se encuentra alejado de la talla promedio encontrada en el presente estudio $(48 \mathrm{~cm})$. En el presente trabajo, se evidencia, entonces, un patrón de dimorfismo sexual que parece ser común en los escualiformes y previamente reportado por Yano y Tanaka (1988). Este patrón se observó en Squalus megalops por Braccini et al. (2006). Este autor indica que frecuentemente se observa un dimorfismo sexual en la talla de tiburones y que, además, es más evidente en especies vivíparas, pues las hembras necesitan mucha más energía para ser empleada en el proceso reproductivo (útero, gónadas y embriones) y, por ende, existe una fuerte presión de selección hacia una talla más grande. Así mismo Fischer et al. (2006), reportaron tallas mayores para hembras con respecto a los machos en Squalus mitsukurii.

Se encontró que los machos pesaron significativamente menos que las hembras de la misma talla, probablemente debido a los mismos factores anteriormente mencionados relacionados con los aspectos reproductivos.

Se determinó el tipo de crecimiento en S. cubensis tanto en hembras como en machos (ver figura 3A y 3B). Los resultados son indicativos de un crecimiento relativo en peso isométrico. Valores muy cercanos al anterior fueron reportados para Carcharhinus falciformis en el golfo de Tehuantepec, en un estudio realizado sobre su biología reproductiva (Ortiz, 2011). De diferente manera, Filiz y Mater (2002) encontraron que Squalus acanthias exhibe un crecimiento alométrico positivo que indica que su peso se incrementa rápidamente con el crecimiento. Esta relación potencial describe el crecimiento relativo en peso y permite estudiar las variaciones espacio-temporales en el factor de condición fisiológica (Lizama y Ambrosio, 2002). La relación talla-peso en peces se ve afectada por varios factores como las condiciones ambientales, estadio de maduración gonadal, sexo, condición estomacal, estación del año, población y diferencias entre especies (Oliveira et al., 2012).

Dicha relación muestra que la ganancia que tiene el organismo en peso es directamente proporcional con la ganancia que tiene en longitud. Las diferencias o similitudes respecto al valor de "b", además de ser atribuidas a factores intrínsecos (genéticos) de las especies, se relacionan también con el hábitat y con la época del año. Según Kanaiwa, Kimoto y Yokawa (2011), la estacionalidad podría ser uno de los factores que afectan estas relaciones entre la talla y el peso de los individuos. Además, Marcano (1990) coincide en este aspecto y menciona el estado de explotación de los recursos como otra posible causa de variación, debido a que las poblaciones son cada vez más afectadas por la utilización desmesurada de artes de pesca poco selectivas que tienen un impacto significativo sobre las especies.

La relación entre las variables de talla y peso ha sido analizada para otros elasmobranquios (Filiz y Mater, 2002; García de la Rosa, Sánchez y Prenski, 2004; Ortiz, 2011). Para S. cubensis no existen trabajos similares que hayan analizado la relación talla - peso, esto se debe, probablemente, a la dificultad de la toma de datos en el campo ya que a veces resulta difícil obtener registros 
de peso de los tiburones. No obstante, para el presente trabajo se contó con un parque de pesca habilitado para realizar dichas mediciones. A lo anterior se le debe sumar el hecho de que esta es una especie de profundidad que no es capturada comúnmente en las faenas de pesca que se practican en la zona. Recientemente se realizó un estudio de los aspectos reproductivos para esta especie, en el que se hace referencia a la relativa alta abundancia $S$. cubensis en la zona del Golfo de México, sin embargo, apelan a las dificultades asociadas al muestreo de los ambientes profundos que impiden conocer más sobre aspectos taxonómicos y biológicos de los tiburones de profundidad (Jones et al., 2013).

En cuanto a la distribución batimétrica de las capturas, Panamá y Honduras (el primero por tener una mayor extensión de línea de costa y el segundo por tener una plataforma continental más ancha) reunieron las condiciones ideales para obtener la mayor cantidad de los ejemplares capturados sobre la plataforma y talud continental del Caribe centroamericano. La mayor cantidad de individuos fueron capturados a profundidades que van desde los 250 hasta los 950 $\mathrm{m}$ en las costas panameñas, en donde ya previamente se había registrado la ocurrencia de $S$. cubensis como especie nativa (Monzini, 2006). La figura 5, indica que la mayor cantidad de lances fueron realizados a profundidades entre los 200 y $400 \mathrm{~m}$ en toda la región. Estas capturas a esas profundidades coinciden con los niveles subtermoclinos, es decir, por debajo de la isoterma de $20^{\circ} \mathrm{C}$ (figura 6). Según el presente estudio, estos individuos se distribuyen fundamentalmente en un estrato donde la temperatura oscila entre los $17^{\circ} \mathrm{C}$ y los $12^{\circ} \mathrm{C}$. Por otro lado, pocos de ellos se capturaron a profundidades donde la temperatura era inferior a $10^{\circ} \mathrm{C}(6 \%)$ o superior a los $20^{\circ}$ (menos del 1\%).

A S. cubensis se le identifica como una especie que forma grandes cardúmenes que nadan en aguas profundas y nunca en aguas superficiales. Los individuos de esta especie se localizan en áreas con fondos fangosos de la plataforma y talud continental, desde 60 hasta $380 \mathrm{~m}$ de profundidad (Compagno, 1984). Sin embargo, los individuos capturados en esta campaña se ubicaron entre los $150 \mathrm{~m}$ hasta los $900 \mathrm{~m}$, sobrepasando la profundidad antes mencionada y los $400 \mathrm{~m}$ reportados como profundidad máxima por Espinosa et al. (2004). Nion (1999) indica que las especies pertenecientes a Squalus son más abundantes en la parte exterior de la plataforma continental en profundidades entre los 50 y $200 \mathrm{~m}$.

Los tiburones de aguas profundas son muy vulnerables a la sobrepesca, ya que, a diferencia de otras especies de tiburones de aguas someras, presentan algunos parámetros de historia de vida limitantes, tales como una madurez tardía, gestación prolongada, baja fecundidad y bajas tasas de crecimiento (Yano y Tanaka, 1989). Aún existe poca información sobre los tiburones de aguas profundas (Kyne y Simpfendorfer, 2007). Para el Caribe de Centroamérica, esta información preliminar sobre proporción de sexos, estructuras de tallas y la relación peso-talla de $S$. cubensis resulta un hallazgo importante, pues es la primera vez que se documenta una captura de esta especie que permite estimar estas variables. La información recabada debe ser complementada con estudios sobre edad, crecimiento, maduración y biología reproductiva, pues esta resulta esencial para conocer acerca de su dinámica poblacional. Se debe tener presente que los tiburones son organismos muy importantes dentro de los ecosistemas marinos, porque forman parte esencial de los niveles tróficos superiores, donde contribuyen a mantener un equilibrio dentro de la comunidad (Pequeño y Lamilla, 1997). 
UNICIENCIA Vol. 31, No. 2, pp. 69-82. Julio-diciembre, 2017.

URL: www.revistas.una.ac.cr/uniciencia

ISSN Electrónico: 2215-3470

Email: revistauniciencia@una.cr

DOI: $\underline{\text { http://dx.doi.org/10.15359/ru.31-2.5 }}$

\section{Agradecimientos}

Este trabajo se llevó a cabo gracias al apoyo económico brindado por la Universidad Nacional. Nuestro agradecimiento al Lic. Mario González Recinos, Director de OSPESCA, quien muy amablemente nos permitió utilizar las bases de datos, y a todo el personal científico y tripulación del B/I Miguel Oliver, de la Secretaría General del Mar de España, que participaron en la Campaña de Investigación P esquera Centroamérica- Caribe 2011.

\section{Referencias}

Alvarado, J. J., Solís-Marín F. A. y Ahearn. C. (2008). Equinodermos (Echinodermata) del Caribe Centroamericano. Revista Biología Tropical, 56(3), 37-55.

Álvarez-León, R. y Rey-Carrasco, I. (2003). Fauna extraída en la exploración del barco M/N “Vikheim” al noroeste del Caribe colombiano. Revista Biología Tropical, 51(2), 551-554.

Arocha, F., Arocha, O. y Marcano, L. A. (2002). Observed shark by catch from the from venezuelan tuna and swordfish fishery from 1994 through 2000. Collective Volume of Scientific Papers, 54(4), 1123-1131.

Baum, J. K., Ransom, A. M., Kehler, D. G., Worm, B., Harley, S. J. y Doherty, P. A. (2003). Collapse and Conservation of Shark Populations in the Northwest Atlantic. Science, 299, 389-392. http://dx.doi. org/10.1126/science.1079777

Braccini, J. M., Gillanders, B. M. y Walker T. I. (2006). Notas sobre la estructura poblacional del tiburón Squalus megalops en el sureste de Australia. Ciencias Marinas, 32(4), 705-712.

Castillo-Géniz, J. L., Márquez-Farias, J. F., Rodriguez de la Cruz, M. C., Cortés, E. y Cid del Prado, A. (1998). The Mexican artisanal shark fishery in the Gulf of Mexico: towards a regulated fishery. Marine and Freshwater Research, 49(7), 611 - 620. http://dx.doi.org/10.1071/MF97120

Compagno, J. V. (1984). FAO species catalogue. Vol 4. Sharks of the World. An annotated and illustrated catalogue of shark species known to date. Part I. Hexanchiformes to Lamniformes. Rome, Italy: FAO Fish Synop.

Compagno, J. V., Dando, M. y Fowler, S. (2005). Sharks of the World. London, England: Collins Press.

Cortés, E. (2004). Life history patterns, demography, and population dynamics. En J. C. Carrier, J. A. Musick \& M. R. Heithaus (Eds), Biology of Sharks and their Relatives (pp. 449-469). USA: CRC press.

Cortés, J. (2007). Coastal morphology and coral reefs: Chapter 7. En J. Bundschuh \& G. E. Alvarado (Eds.) Central America: Geology, Resources and Hazards (Vol. 1.), (pp. 185-200). London: Taylor \& Francis.

Daniel, W. W. (2012). Distribución ji-cuadrada y análisis de frecuencias. Capítulo 12. En W. W Daniel (Ed), Bioestadística: Base para el análisis de las ciencias de la salud (4ta edición), (71-583). México: Limusa Wiley.

Espinosa, P. H., Castro-Aguirre, J. L y Huidobro, C. L. (2004). Listados faunísticos de México. IX Catálogo sistemático de tiburones. (Elasmobranchii: Selachimorpha), (pp. 103-104). Instituto de Biología. Universidad Autónoma de México.

FAO. (2006). Organización de las Naciones Unidas para la Alimentación y la Agricultura. Recuperado de http://www.fao.org/fishery/countryprofiles/search/es

Filiz, H., y Mater, S. (2002). A Preliminary Study on Length-Weight Relationships for Seven Elasmobranch Species from North Aegean Sea, Turkey. Su Ürünleri Dergisi, 19(3).

Fischer, A. F., Veras, D. P., Hazin F. H. V., Broadhurst, M. K., Burgess, G. H. y Oliveira. P. G. V. (2006). Maturation of Squalus mitsukurii and Cirrhigaleus asper (Squalidae, Squaliformes) in the southwestern equatorial Atlantic Ocean. Journal of Applied Ichthyology, 22, 495-501. http://dx.doi. org/10.1111/j.1439-0426.2006.00749.x 
ISSN Electrónico: 2215-3470

DOI: http://dx.doi.org/10.15359/ru.31-2.5
UNICIENCIA Vol. 31, No. 2, pp. 69-82. Julio-diciembre, 2017.

URL: www.revistas.una.ac.cr/uniciencia Email: revistauniciencia@una.cr

García de la Rosa, S., Sánchez, F. y Prenski, B. (2004). Caracterización biológica y estado de explotación del tiburón espinoso (Squalus acanthias). En Sánchez, R. \& Bezzi, S. (Eds), El mar argentino y sus recursos pesqueros. Biología y evaluación del estado de explotación (Tomo 4). Mar del Plata: Publicaciones especiales INIDEP.

Hazin, F. H. V., Fischer, A. F., Broadhurst, M. K., Veras, D., Oliveira, P. G. y Burgess, G. H. (2006). Notes on the reproduction of Squalus megalops off northeastern Brazil. Fisheries Research, 79, 251-257. http://dx.doi.org/10.1016/j.fishres.2006.04.006

Jackson, J. B. C y D’ croz, L. (1997). The ocean divided. En A. G Coates (Ed.), Central America: A Natural and Cultural History (pp. 38-71). New Haven. USA: Yale University.

Jones, L. M., Driggers III, W. B., Hoffmayer, E. R., Hannan, K. M., y Mathers, A. N. (2013). Reproductive biology of the Cuban dogfish in the northern Gulf of Mexico. Marine and Coastal Fisheries, 5(1), 152-158. http://dx.doi.org/10.1080/19425120.2013.768572

Kanaiwa, M., Kimoto, A. y Yokawa, K. (2011). Standardized CPUE of striped marlin in north western Central Pacific Ocean by using GLM. ISC/11/BILLWG - 2/06.

Kiraly, S. J., Moore, J. y Jasinski, P. H. (2003). Deepwater and Other Sharks of the U.S. Atlantic Ocean. Exclusive Economic Zone. Marine Fisheries Review, 65(4), 1-20.

Kyne, P. M. y Simpfendorfer, C. A. (2007). A collation and summarization of available data on deep water chondrichthyans: biodiversity, life story and fisheries. A report prepared by the IUCN SSC Shark Specialist Group for the Marine Conservation Biology Institute Peter M.

Lizama, M. D. L. A. P., y Ambrosio, A. M. (2002). Condition factor in nine species of fish of the Characidae family in the upper Paraná river floodplain, Brazil. Brazilian Journal of Biology, 62(1), 113-124. http://dx.doi.org/10.1590/S1519-69842002000100014

Marcano, L. A. (1990). Análisis de la situación de la industria de la pesca de arrastre durante el periodo 1985-1989. En Resultados de talleres sobre la pesca en Venezuela, (pp. 25-52). Caracas, Venezuela: MAC-DGSPA.

Márquez, J. F. (2002). Análisis de la pesquería de tiburón de México (Tesis de maestría). Universidad de Colima, México.

Menni, R. C. y Lucifora, L. O. (2007). Condrictios de la Argentina y Uruguay. Lista de trabajo. ProBiota, FCNyM, UNLP, Serie Técnica-Didáctica, 11, 1-15. La Plata, Argentina.

Monzini, J. (2006). Squalus cubensis. The IUCN Red List of Threatened Species 2006: e.T61416A12476876. Recuperado de http://dx.doi.org/10.2305/IUCN.UK.2006.RLTS.T61416A12476876

Nelson, J. S., Grande, T. C. y Wilson, M. V. H. (2016). Class Chondrichthyes. En J. Nelson (Ed), Fishes of the world. 5th edition, (pp. 66), New Jersey. EE.UU.: John Wiley and sons.

Nion, H. (1999). La pesquería de tiburones en Uruguay con especial referencia al cazón (Galeorhinus galeus, Linnaeus 1758). En R. Shotton (Ed), Case studies of the management of elasmobranch fisheries. FAO Fisheries Technical Paper, N. ${ }^{\circ}$ 378, part 1. Rome: FAO.

Oliveira, M. R., Costa, E. F., Araújo, A. S., Pessoa, E. K. R., Carvalho, M. M., Cavalcante, L. F. M. y Chellappa, S. (2012). Sex ratio and length-weight relationship for five marine fish species from Brazil. Journal of Marine Biology \& Oceanography, 1(2). http://dx.doi.org/10.4172/2324-8661.1000103

Ortiz, P. T. (2011). Biología reproductiva del tiburón aleta de cartón Carcharhinus falciformis (Muller y Henle, 1839) en la costa de Chiapas, México (Tesis de Licenciatura). Universidad del Mar, Campus Puerto Ángel, Oaxaca, México.

Páramo, J., Wolff, M. y Saint-Paul, U. (2012). Deep-sea fish assemblages in the Colombian Caribbean Sea. Fisheries Research, 125-126, 87-98. http://dx.doi.org/10.1016/j.fishres.2012.02.011 
UNICIENCIA Vol. 31, No. 2, pp. 69-82. Julio-diciembre, 2017.

ISSN Electrónico: 2215-3470

URL: www.revistas.una.ac.cr/uniciencia

DOI: $\underline{\text { http://dx.doi.org/10.15359/ru.31-2.5 }}$

Email: revistauniciencia@una.cr

Pequeño, G. y Lamilla, J. (1997). Las pesquerías de condrictios en Chile: Primer análisis. Biología Pesquera, 26, 13-24.

Rivero, L. H. (1936). Some new, rare and little-known fishes from Cuba. Boston Society of Natural History, 41(4), 41- 76.

SURFER 11. (2012). Surfer ${ }^{\circledR}$ Contouring and 3D Surface Mapping for Scientists and Engineers, version 11 www.GoldenSoftware.com. Colorado, EE. UU.: Golden Software.

Viana, S. T., Carvalho, M. R., y Gomes, U. L. (2016). Taxonomy and morphology of species of the genus Squalus Linnaeus, 1758 from the Southwestern Atlantic Ocean (Chondrichthyes: Squaliformes: Squalidae). Zootaxa, 4133(1), 1. http://dx.doi.org/10.11646/zootaxa.4133.1.1

Yano, K. y Tanaka, S. (1988). Size at maturity, reproductive cycle, fecundity, and depth segregation of the deep sea Squaloid sharks Centroscymnus owstoni and C. coelolepis in Suruga Bay, Japan. Nippon Suisan Gakkaishi, 54: 167-174. http://dx.doi.org/10.2331/suisan.54.167

Yano, K. y Tanaka, S. (1989). Hermaphroditism in the lantern shark, Etmopterus unicolor (Squalidae, Chondrichthyes). Japanese Journal of Ichthyology, 36(3), 338- 345

\section{@) $\odot \Theta \Theta$}

Proporción sexual, relación talla-peso y distribución geográfico-batimétrica de Squalus cubensis (SQUALIFORMES:

SQUALIDAE) en la costa Caribe de Centroamérica (Rosario Benavides-Morera y otros) por Revista Uniciencia se encuentra bajo una Licencia CreativeCommons Atribución-NoComercial-SinDerivadas 3.0 Unported. 